\title{
Contemporary hysteroscopic methods for female sterilization
}

\author{
Roger D. Smith * \\ Department of Obstetrics and Gynecology, University of Michigan Medical Center, Ann Arbor, USA
}

\section{A R T I C L E I N F O}

\section{Article history:}

Received 24 June 2009

Accepted 20 July 2009

\section{Keywords:}

Adiana

Essure

Hysteroscopy

Hysteroscopic sterilization

Sterilization

\begin{abstract}
A B S T R A C T
A permanent contraceptive method that avoids abdominal incisions and general anesthetic should be safer than sterilization by laparoscopy or laparotomy. In theory, the transcervical route ought to be ideal for female sterilization. However, past attempts have not seen widespread success, and contemporary efforts demonstrate that challenges to the creation of an ideal transcervical sterilization technique continue to exist. After 6 years of use, clinical data and real-world experience indicate that the Essure permanent birth control system is a viable option. Efficacy of $99.74 \%$ has been demonstrated. Adverse effects and risks are low. Patient satisfaction is high. Successful placement is observed in worldwide marketing. It can be placed in the office setting, which offsets the relatively high cost of the device. Recent data suggest that patients and surgeons are choosing hysteroscopic sterilization over laparoscopic and postpartum sterilization. Adiana emerged in 2009 as a second hysteroscopic sterilization option. Challenges continue to exist for transcervical sterilization. Compliance with post-procedure confirmation imaging is not universal. Real-world contraception failures are seen in a setting of protocol noncompliance. However, extrapolation of the failure rates in real-world use seems to be comparable with other laparoscopic and abdominal sterilization methods.

(c) 2009 Published by Elsevier Ireland Ltd. on behalf of International Federation of Gynecology and Obstetrics.
\end{abstract}

\section{Introduction}

Female sterilization is the single most prevalent method of contraception in the world [1]. United Nations data for 2007 show that of the 1.1 billion partnered women practicing contraception, $20 \%$ were using female sterilization. The next most prevalent modern methods were intrauterine devices (16\%), oral contraceptives (9\%), condoms (6\%), male sterilization (3\%), and injectable hormones (3\%). Permanent female contraception routinely entails exposing women to abdominal incisions, and thus to the related risks of surgery and anesthesia. Although they are generally extremely safe, the traditional methods for sterilization in women carry risks for major complications, including mortality [2]. A permanent contraceptive method that avoids abdominal incisions and general anesthesia should be safer than sterilization by laparoscopy or laparotomy. In theory, the transcervical route ought to be ideal for female sterilization. However, past attempts have not seen widespread success, and contemporary efforts demonstrate the challenges remaining for an optimal method to accomplish tubal occlusion transcervically.

\subsection{History: Electrosurgical energy}

Cooper [3] presented a detailed history of transcervical sterilization in 1992. First attempted in the 1920s [4], hysteroscopic

\footnotetext{
* Department of Obstetrics and Gynecology, University of Michigan Medical Center, Women's Hospital, Ann Arbor 48109, USA.

E-mail address: rogersmi@umich.edu.
}

application of electrocautery to cause infertility saw a resurgence in the 1970s. Quinones et al. [5] performed over 1200 hysteroscopic sterilizations with tubal endocoagulation and observed a bilateral occlusion rate of $80 \%$. No pregnancies occurred after 1 year of observation in 513 patients whose hysterosalpingogram had shown bilateral occlusion. However, of 423 patients monitored for 5 years in whom the hysterosalpingogram had demonstrated occlusion, 3.8\% eventually became pregnant [3]. A subsequent collaborative series showed a $3.2 \%$ pregnancy rate among patients with a hysterosalpingogram showing occlusion, and a 3.2\% major complication rate, including a death after bowel injury [6].

\subsection{History: Mechanical devices}

Many mechanical devices for tubal occlusion have been proposed or tried in animals and humans, with limited success. Tube-occluding substances have included hydrogel/nylon (P-block) [7]; silicone Ovabloc [8]; polyethylene [9]; nylon [10]; and polytetrafluoroethylene [11].

\subsection{History: Chemical}

Quinacrine hydrochloride has been studied extensively. Quinacrine can be instilled blindly or hysteroscopically. It causes inflammation and subsequent scar tissue formation within the fallopian tubes. Over 100000 quinacrine sterilization procedures have been performed worldwide, but the technique is marred in controversy [12]. 


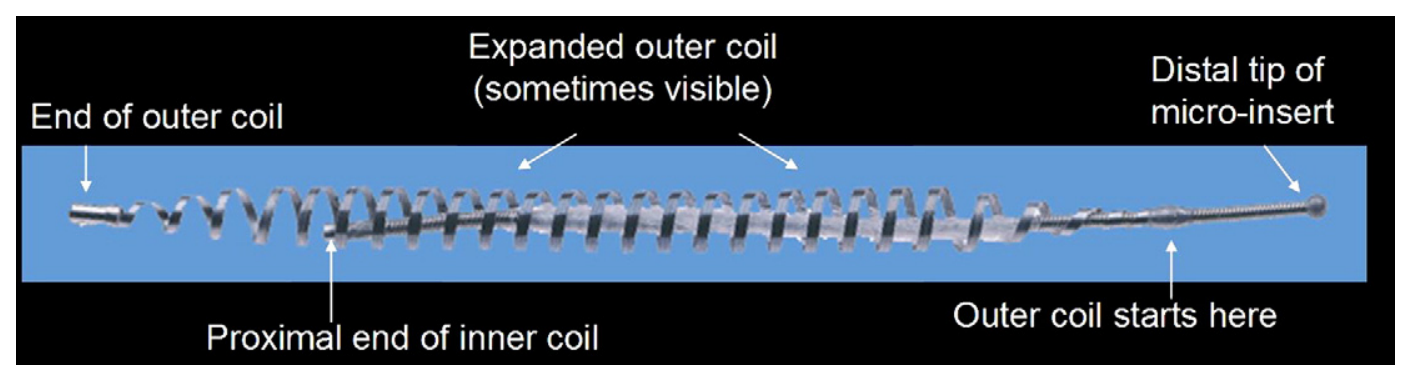

Fig. 1. Essure design.

\subsection{Lessons from the past}

Many of these methods are available options for women worldwide. However, none sees widespread use. Kerin [13] cites improvements in the design and application of fallopian tube cannulation devices used for infertility treatments as the foundation for the creation of successful transcervical sterilization techniques. Catheter technology has advanced adequately to be able to reliably negotiate an occluding substance into the fallopian tubes. Cooper [3] identifies that, in the past, mechanical devices failed because they migrated or were expelled too frequently. He emphasizes that in order to anchor an implant for tubal occlusion, one must take advantage of the less-compliant uterine portion of the fallopian tube. Some past failures occurred because the implanted medium did not result in complete tubal occlusion. Some devices were temporary; others did not adhere closely enough to the tubal endothelium to provide adequate occlusion.

\section{Essure system}

The Essure Permanent Birth Control system (Conceptus; Mountain View, CA, USA) was marketed first in Australia and Singapore. Approval by the European Union followed in 2001, and by the United States Food and Drug Administration late in 2002. It is now also available across North, Central, and South America, and in parts of Asia and the Middle East.

\subsection{Design}

The micro-insert is made of a flexible stainless steel inner coil, surrounded by a dynamic outer coil composed of nickel titanium (Nitinol). Polyethylene terephthalate (PET) fibers run along and through the inner coil. (Fig. 1) In its expanded form, the implant is about $4 \mathrm{~cm}$ long and up to $2 \mathrm{~mm}$ in diameter. The disposable delivery system includes a nitinol delivery wire, a release catheter, a hydrophilic delivery catheter, and a handle with mechanisms to retract the release catheter and the delivery catheter. The materials that compose the micro-insert have a long history of use in medical and surgical devices. For example, Dacron (Invista; Charlotte, NC, USA), a polyester made of PET fibers, has been used in suture, grafts, and stents for about 40 years. Tubal occlusion occurs because of tissue reaction toward the presence of the PET fibers. The stainless steel-nickel titanium coil serves as an anchor within the utero-tubal junction, which keeps the PET fibers in the proper location for tissue in-growth to occur following placement. The PET fibers elicit a benign local inflammatory response, which peaks between 2 and 3 weeks after placement. This inflammatory response gradually resolves over a 10-week period.

\subsection{Placement}

Placement of the Essure micro-coils requires a rigid hysteroscope with a 5-Fr operating channel. Most commercially available hysteroscopes have a $5.5 \mathrm{~mm}$ outer diameter operating sheath with inflow and outflow ports for a fluid distending medium. Warmed normal saline is typically used for distension. After performing diagnostic hysteroscopy, and confirming that bilateral placement is possible, the catheter is introduced under direct visualization into a fallopian tube. Delivery and release catheters are retracted, and the coil expands to anchor itself in the tube. After detaching the implant from a guide wire, the micro-coil spans the utero-tubal junction. Part of the microcoil trails into the uterine cavity, and the rest of the coil remains within the fallopian tube (Fig. 2).

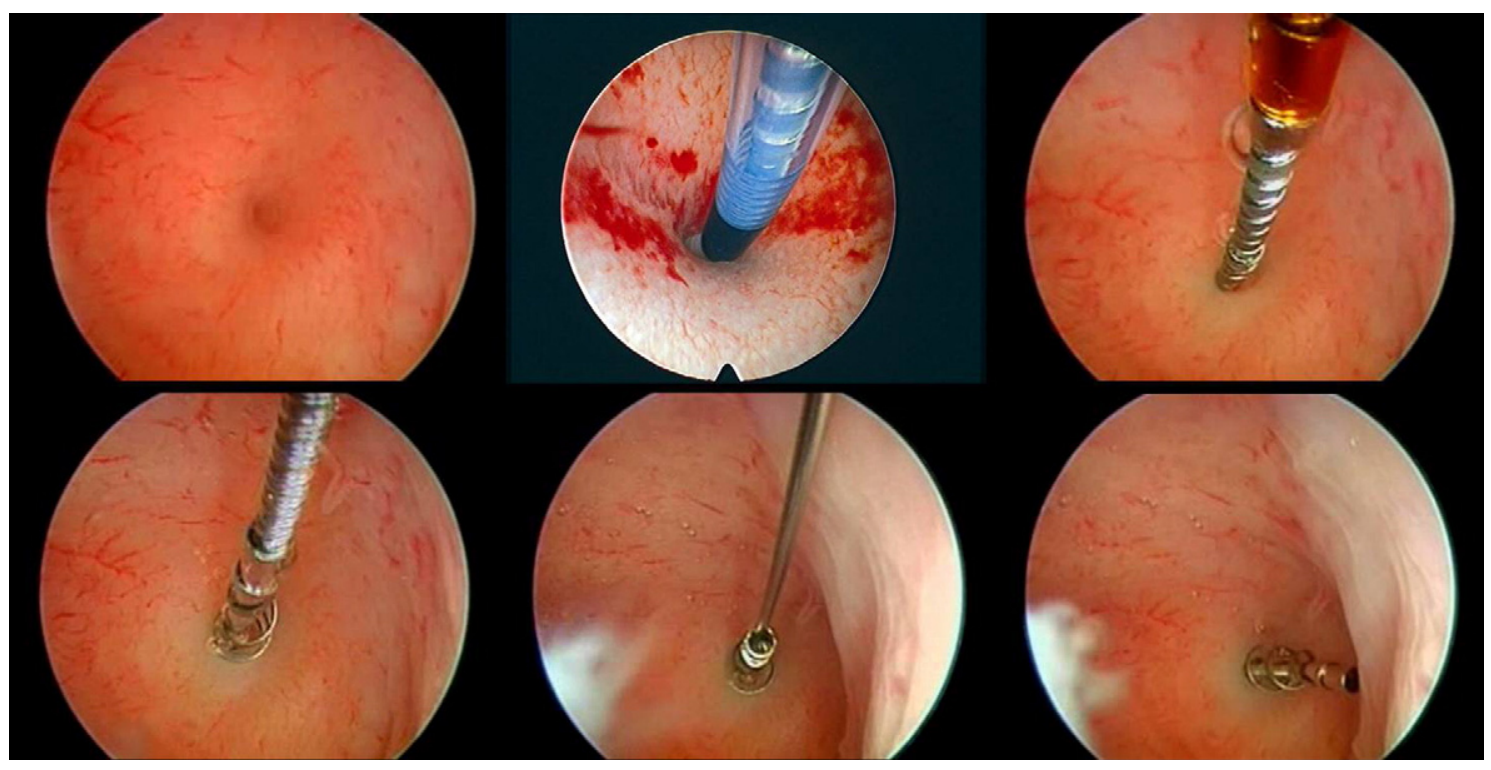

Fig. 2. Placement procedure. 


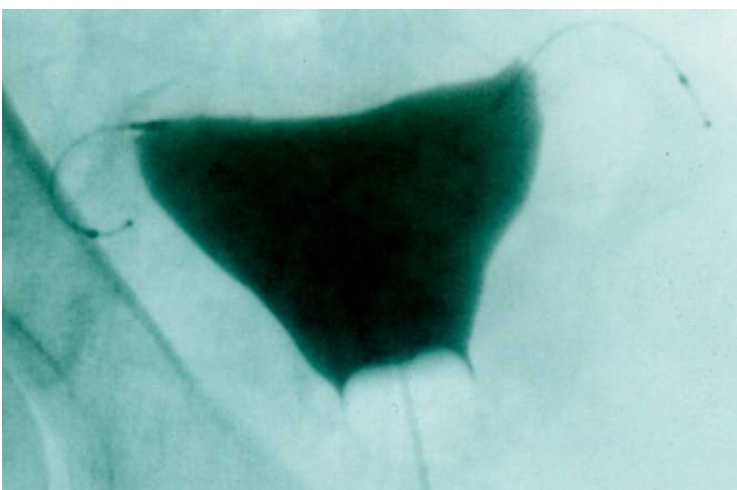

Fig. 3. Hysterosalpingogram showing proper implant location and bilateral occlusion.

\subsection{Confirmatory hysterosalpingography}

After placement, the patient must practice reliable contraception for at least 3 months. Sterilization does not occur until the PET fibers cause local tissue in-growth, which occludes the tubes. Hysterosalpingography (HSG) is performed 3 months after placement to demonstrate proper coil location across the utero-tubal junction, and to demonstrate tubal occlusion. A patient should not rely on the system for contraception until she has a hysterosalpingogram that shows that these criteria have been met (Fig. 3).

\section{Essure literature review}

Over 2500 patients have undergone the procedure as part of a clinical trial. The subsequent review focuses on the Phase II and Phase III (Pivotal) FDA clinical trials [14,15].

\subsection{Phase II and pivotal trial summary}

Together, the trials enrolled 745 women (227 Phase II, 518 Pivotal) in prospective multicenter, international, single-arm trials conducted between 1998 and 2001. All of the patients were parous, with a mean age of 32 years. The procedures were performed under paracervical block, some with intravenous sedation, and some with non-steroidal anti-inflammatory drugs (NSAIDs). Office and telephone follow-up occurred at frequent intervals to assess for adverse events, and to evaluate patient satisfaction. All of the patients practiced alternative contraception until they underwent HSG 3 months after their procedure. The length of time of the procedure ranged from 13 to 18 minutes. The recovery time ranged from 30 to 44 minutes.

\subsection{Placement success}

In the Pivotal Trial [15], the surgeons encountered uterine cavity pathology that precluded a placement attempt in $2 \%$ of the patients. If one includes all patients who intended to undergo the procedure in the denominator (intent-to-treat group), bilateral placement was achieved in $86 \%$ of patients in the first procedure. Many patients underwent a second procedure, which brought bilateral placement up to $90 \%$. The clinical trials and this author's experience show that several factors can contribute to placement failure. Tubal spasm, a frequently-encountered hindrance to cannulating a tube, can be overcome by warming the distension medium, and by administering an NSAID preoperatively. Preexisting tubal stenosis can be encountered. Extremely lateral tubes and a very retroverted uterus make the path to the tubal ostium difficult. A visual field compromised by bleeding can be avoided by preparing the endometrium with hormonal contraceptives, and by avoiding unnecessary cervical manipulation with uterine dilators or uterine sounds. Most multiparous cervices do not require dilation prior to passing a 5.5mm hysteroscope.

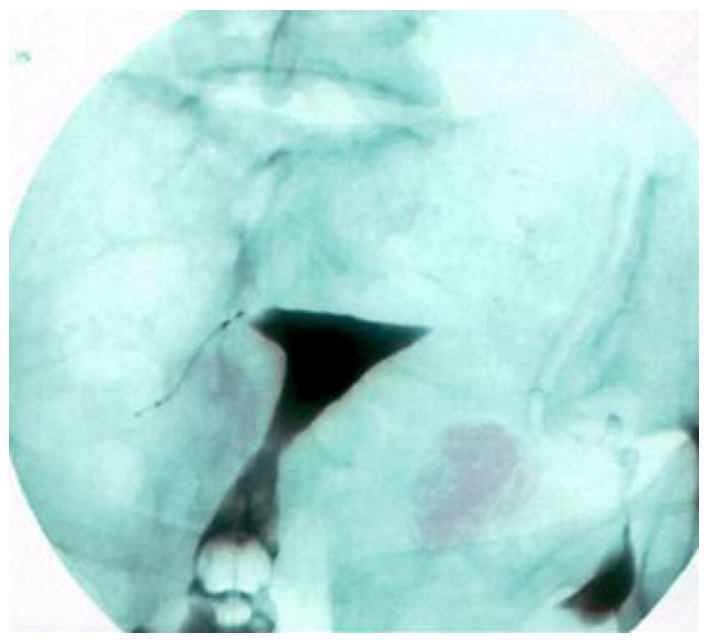

Fig. 4. Expulsion of implant from left side.

\subsection{Adverse events}

Adverse events or side effects occurred in $3 \%$ of patients on the day of the procedure. These included vasovagal responses, hypervolemia, cramping, nausea, and vaginal spotting. Except for one patient who was observed overnight for a reaction to narcotics, all of the patients were discharged home on the same day. During the first year of observation, the following adverse effects were reported most commonly: back pain (9\%); abdominal cramps (3.8\%); dysmenorrhea (2.9\%); and pelvic pain (2.5\%). A comparison with traditional female sterilization techniques emphasizes the better safety profile observed by avoiding abdominal incision. Major complications-conversion to laparotomy, blood transfusion, febrile morbidity, rehospitalization-occur in $0.9 \%$ to $1.6 \%$ of traditional female sterilization surgeries [2]. A mortality rate of 1-4 per 100000 has been estimated. In the Pivotal Trial, perforation through the fallopian tube occurred in $1 \%$ of patients. Perforation is usually asymptomatic, recognized only at HSG. Hysteroscopic sterilization also compares favorably with vasectomy. Minor complications-wound infection, bleeding, hematoma, granuloma formation, epididymitishave been observed in $5 \%$ to $10 \%$ of vasectomy procedures. Late sequelae include chronic testicular pain in almost $1 \%$ of patients [16].

\subsection{Interpretation of the hysterosalpingogram}

Expulsion of the micro-coil from the uterus, or migration of the implant from an acceptable location within the tube, occurred in 3\% of patients. Fig. 4 shows expulsion of the micro-coil from the left side. HSG demonstrates tubal patency in about $4 \%$ of patients at 3 months, despite proper implant location. Fig. 5 shows tubal patency on the right side. Some

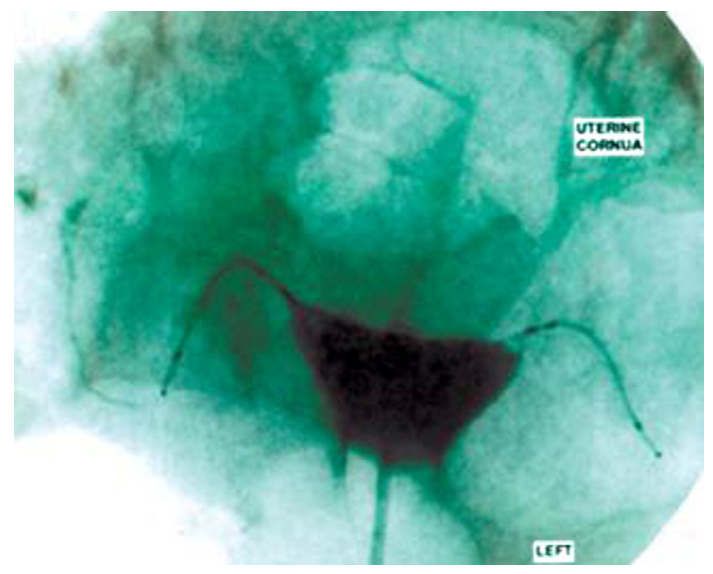

Fig. 5. Tubal patency on right. 
patients display slower rates of tissue in-growth in response to the PET fibers within the micro-coil. At 6-month HSG, all patients with proper coil location demonstrated occluded tubes. Because proper micro-coil location has correlated with tubal occlusion in clinical trials, some surgeons avoid performing HSG when alternative imaging (flat plate, sonography) confirms proper location. Outside of the United Sates, post-Essure HSG is not routinely performed in all countries.

\subsection{Reliance rate}

Requiring HSG after hysteroscopic sterilization not only sets this new procedure apart from laparoscopic sterilization and laparotomy sterilization, it also entails some new definitions. The "reliance rate" is defined with the denominator of patients in whom bilateral placement is achieved. The numerator is patients who undergo HSG and show proper micro-coil location and tubal occlusion. This number demonstrates that approximately $3 \%$ of the time, properly placed coils migrate or are expelled after initial placement.

\subsection{Summary}

A summary of the data from the trials that led to FDA approval of Essure points out several challenges to hysteroscopic sterilization. Two percent of those who intended to use Essure had no attempt at coil placement because of uterine cavity pathology. After one procedure, $86 \%$ bilateral placement was achieved. After a second procedure, bilateral placement increased to $90 \%$. Some patients had to wait up to 6 months until they could rely on the procedure. Three percent of patients could never rely on the procedure because of improper microimplant location. Finally, if one defines the intent-to-treat group as all the patients who undergo a hysteroscopy to have Essure coils placed, $13 \%$ of them ultimately had to choose a different method of contraception. Failure to achieve bilateral device placement remains the most common factor necessitating an alternate contraception choice.

\subsection{Post-approval placement trials}

Several clinical trials demonstrate improved bilateral placement rates. Kerin et al. [17] reported on 109 patients recruited after the Pivotal study. With more experience, and a redesigned outer delivery catheter, he achieved $98 \%$ bilateral placement after one procedure. Mino et al. [18] reported 96\% bilateral placement in one procedure, and $98 \%$ after a second. The FDA linked its approval of Essure to subsequent enrollment of 800 patients in a clinical trial with 40 new surgeons performing the procedure. This Post-Approval Study, not yet published, demonstrated $94.6 \%$ bilateral placement.

\section{Essure efficacy}

Discussion of contraceptive effectiveness points out another way that the unique features of this hysteroscopic sterilization procedure differentiate it from traditional female sterilization. "Effectiveness" is defined in clinical trials as a lack of pregnancies in women who have achieved bilateral placement, and have had a confirmatory hysterosalpingogram showing proper micro-coil location and occluded tubes. When patients have met the criteria to rely on Essure, no known pregnancy has occurred in clinical trials to date. Using an adjusted indirect method to calculate effectiveness, similar to that in the CREST trial [2], 5-year data show an efficacy rate of $99.74 \%$.

\subsection{Effectiveness: Consumer use}

Through October 2008 there had been 258 Essure-related pregnancies reported to Conceptus. Scrutiny of these pregnancies shows that they have occurred in a variety of patients who did not meet the criteria to be able to rely on Essure. Levy et al. [19] analyzed a subset of these pregnancies and described the factors associated with failure. Patient non-compliance represents the most common factor. Some patients did not practice alternative contraception during the 3 months following placement. Some patients did not present for HSG. There were some luteal phase pregnancies. A small number of pregnancies were attributed to "physician non-compliance." That is, a physician recommended reliance on Essure when the defined criteria were not met. Particularly troublesome is that in 18 of 64 pregnancies, review showed that the hysterosalpingogram had not been interpreted correctly. A specific protocol has now been developed for the performance of and interpretation of the post-placement hysterosalpingogram. Levy points out that, especially when the surgeon who places the Essure micro-coils is not involved in the HSG, it is important to make sure that the HSG team is familiar with the protocol. This author is continually surprised at the number of ways a post-Essure hysterosalpingogram can be a challenge to interpret.

\subsection{Effectiveness: Extrapolation}

An estimate of the number of procedures performed worldwide can be assumed from the number of procedure kits shipped. Through October 2008, about 242263 units had been shipped. One can extrapolate a commercial-use failure rate from the known numbers of pregnancies and the procedures performed. A total of 258 pregnancies among 242263 procedures suggests a failure rate of about 1.06 per 1000. Real-world use of Essure demonstrates that patients who intend to use it for contraception will experience pregnancy-whether because of patient or physician non-compliance, or device failure. Traditional sterilization procedures are not routinely followed by HSG. It may be useful for surgeons and patients to compare the rate of pregnancy after Essure with the rate of pregnancy after laparoscopic sterilization. This is at least as favorable as the reported failure in CREST [2].

\section{Essure cost analysis}

In 2008, the Essure system (one pair of micro-coils and disposable insertion devices) cost US $\$ 1300$ in the United States. Although this new technology is expensive, a cost saving is realized in shorter procedure times, and often, in avoidance of the operating room via office placement. A retrospective look at the cost of laparoscopic sterilization in an operating room compared with Essure in an office setting showed Essure to be less expensive [20]. The total cost of a sterilization procedure was US $\$ 2050$ for laparoscopy, and US \$1374 for Essure. Avoiding the operating room eliminated the cost of hospital staff salaries, room time, supplies, and anesthesiology services. The cost of the surgeon's time was slightly less also. Even a comparison of hospital laparoscopic sterilization with Essure performed in an operating room showed some savings via hysteroscopy [21]. A retrospective cohort study of sterilizations showed total costs for laparoscopy and hysteroscopy to be US $\$ 2880$ and US $\$ 2700$, respectively. None of these studies factored in the opportunity cost savings realized by patients enjoying a shorter recovery time and a quicker return to work or family.

\section{Challenges, pitfalls, unique features}

\subsection{Permanency}

Essure sterilization is permanent. Unlike postpartum or laparoscopic tubal ligation, tubal reversal after Essure is not feasible. One can argue whether this represents a reason to avoid this sterilization technique. But, the information is certainly an important part of preoperative counseling.

\subsection{Pregnancy in the presence of micro-coils}

Pregnancy outcomes look favorable when pregnancy occursplanned or unplanned-in the presence of the Essure micro-coils. 
Healthy term pregnancies have been observed when Essure has been used to treat hydrosalpinx with subsequent in vitro fertilization [22]. Unpublished data regarding the pregnancies reported to Conceptus suggest spontaneous abortion rates and pregnancy complication rates are not higher in the presence of the microcoils.

\subsection{Uterine instrumentation after Essure}

Blind uterine instrumentation after Essure might displace a microcoil. Currently, it is prudent to perform any cavity instrumentation after Essure via direct hysteroscopic visualization. This is a topic ripe for investigation. Properly-placed coils become encapsuled by endometrial tissue [23]. The fear of disrupting the micro-coils and causing harm or altering contraceptive efficacy may turn out to be unwarranted.

\subsection{Endometrial ablation}

There is considerable experience with concomitant Essureendometrial ablation procedures, and with endometrial ablation performed after Essure is confirmed by HSG [24-26]. Concern continues over the application of electrosurgical energy in the presence of Essure micro-coils. For instance, endometrial resection or ablation, or global ablation via radiofrequency energy (Novasure) poses a theoretical, if not a demonstrable, risk of conducting energy toward the bowel. Thermal global ablation was initially marketed as a viable procedure to perform concomitantly with Essure. Concern over the ability to interpret the post-Essure hysterosalpingogram after endometrial ablation has led to a change in FDA labeling. Essure placement and confirmation should be accomplished prior to endometrial ablation.

\subsection{Alternatives to HSG}

There are several ways to view the confirmatory hysterosalpingogram, which continues to be recommended after Essure. One viewpoint sees hysterosalpingogram as a "test of cure," much like the semen analysis that is routinely done after vasectomy to demonstrate sterility. However, HSG adds to the cost of this procedure and is inconvenient for the patient. One retrospective review of patient compliance in a general clinic population showed that only $12.7 \%$ of patients presented to undergo HSG after the successful bilateral Essure placement [27]. Several alternatives to HSG have been advocated. Since studies to date suggest that all tubes are eventually (at 6 months, if not at 3 months) occluded when the micro-coils are located properly, imaging may only need to demonstrate implant location. Plain films, standard sonography, and contrast infusion sonohysterography have been applied as alternatives to confirmation using HSG. However, studies to date have not been powered to be able to demonstrate that any alternative imaging technique can replace HSG [28-30]. Indeed, one cannot make definite conclusions about exactly what it means to see tubal occlusion or patency using HSG after Essure. Pregnancy after an unconfirmed Essure placement looks like it is exceedingly rare. Commercial use and more studies will shed light on whether HSG is any more necessary after hysteroscopic sterilization than it is after laparoscopic sterilization.

\section{Other hysteroscopic options: Adiana transcervical sterilization system}

This promising procedure causes tubal occlusion through a combination of radio-frequency tubal endocoagulation and the implantation of a silicone matrix [31,32]. Adiana (Hologic; Bedford, MA, USA) received CE marking approval in January 2009, allowing it to be marketed to European Union countries. FDA approval for marketing in the United States followed in July, 2009.

\subsection{Design: Placement}

In the Adiana system the delivery catheter is introduced through a 5 -Fr hysteroscope working channel. Proper depth of placement within the cannulated tube is accomplished with visual cues on the delivery catheter. A bipolar electrode array applies 60 seconds of RF energy (3 Watts) to achieve a temperature of $64^{\circ} \mathrm{C}$. After endocoagulation, a porous cylindrical matrix of silicone $(3.5 \mathrm{~mm}$ long, $1.5 \mathrm{~mm}$ in diameter) is released in apposition within the treated area and the catheter is removed. Tissue in-growth occurs within the matrix, which leads to tubal occlusion. The patient cannot rely on the procedure for contraception until occlusion is confirmed by HSG 3 months after the procedure. Unlike the Essure micro-coils, the Adiana silicone matrix is not radio opaque. Ultrasound has been used to visualize the matrix.

\subsection{Literature}

The Phase III trial had 645 women in the intent-to-treat group [15]. Bilateral placement was achieved in $95 \%$. A total of 570 out 645 (88.4\%) complied with follow-up and were ultimately able to rely on Adiana. Similar to Essure, about 3\% had to wait 6 months to see tubal occlusion demonstrated using HSG. Eleven pregnancies occurred during the first 15 months of postprocedure follow-up. Of these 11, 6 occurred in women who complied with follow-up and were told they could rely on the device. Five pregnancies occurred in patients who experienced placement failure or who did not use alternative contraception prior to undergoing HSG. The 6 pregnancies indicated a 1-year failure rate of $1.1 \%$. Long-term data are not yet available, but the data submitted to the FDA indicates 4 other pregnancies from the group who were told they could rely on Adiana, for a total of 10 . This increases the failure rate to $1.8 \%$ at 2 years.

\subsection{Adverse events}

Patients reported high satisfaction rates for this 12-minute outpatient procedure. Same-day and 1-year adverse events were minor, with the exception of one ectopic pregnancy among the patients instructed to rely on Adiana.

\subsection{Hysterosalpingography}

Three of the 6 pregnancies among compliant patients at 1 year of follow-up are attributed to misinterpretation of the hysterosalpingogram. This underscores the problematic nature of HSG to confirm the ability to recommend reliance after hysteroscopic sterilization. It is not clear whether HSG-alternatives may be viable options after Adiana, as they have been proposed after Essure.

\subsection{Summary}

Long-term data will help define the efficacy of Adiana at preventing pregnancy. It looks like it may be another safe, effective outpatient transcervical sterilization option. However, initial data are disappointing when comparing Adiana with laparoscopic sterilization or to Essure.

\section{Conclusion}

Clinical data and real-world experience indicate that a viable transcervical sterilization technique finally exists. Recent data suggest that patients and surgeons are choosing hysteroscopic sterilization over laparoscopic and postpartum sterilization [33]. The relative safety of hysteroscopic tubal occlusion over methods that require abdominal incision offers a compelling reason for sterilization patients to choose hysteroscopy. The techniques currently available do pose some new challenges. Although the devices are relatively expensive, the ability to 
place the implants in the office setting offers a cost-saving opportunity. This will pave the way for making this long-term birth control method an option worldwide.

\section{Conflicts of interest}

No conflicts of interest to declare.

\section{References}

[1] United Nations Department of Economic and Social Affaires-Population Division. World Contraceptive Use 2007. Wall chart. New York, NY: United Nations Publications; 2008

[2] American College of Obstetrics and Gynecology. ACOG Practice Bulletin. Benefits and Risks of Sterilization. Number 46, September 2003. Int J Gynecol Obstet 2003:83(3):339-50.

[3] Cooper JM. Hysteroscopic sterilization. Clin Obstet Gynecol 1992;35(2):282-97.

[4] Mikulicz-radecki F, Freund A. A new hysteroscope and its practical use in gynecology. [in German]. Z Geburtshilfe Gynakol 1928;92:13-25.

[5] Quinones R, Alvarado A, Ley E. Hysteroscopic sterilization. Int J Gynecol Obstet 1976;14(1):27-34.

[6] Darabi KF, Richart RM. Collaborative study on hysteroscopic sterilization procedures. Preliminary report. Obstet Gynecol 1977;49(1):48-54.

[7] Brundin J. Observations on the mode of action of an intratubal device, the P-block. Am J Obstet Gynecol 1987;156(4):997-1000.

[8] Houck RM, Cooper JM. Hysteroscopic tubal occlusion with formed-in-place silicone plugs. Obstet Gynecol 1982;60(5):641-8.

[9] Hosseinian AH, Lucero S, Kim MH. Hysteroscopic implantation of utero-tubal junction blocking devices. In: Sciarra JJ, Droegemueller W, Speidel JJ, editors. Advances in Female Sterilization Techniques. Hagerstown, MD: Harper \& Row; 1976.

[10] Hamou J, Gasparri F, Scarselli GF, Mencaglia L, Perino A, Quartararo P, et al. Hysteroscopic reversible tubal sterilization. Acta Eur Fertil 1984;15(2):123-9.

[11] Majos MB, Chapman MB. Hysteroscopic tubal sterilization. Obstet Gynecol Clin N Am 2004;31:705-919.

[12] Zipper J, Kessel E. Quinacrine sterilization: a retrospective. Int J Gynecol Obtset 2003;83(Suppl 2):S7-S11.

[13] Kerin JF. New methods for transcervical cannulation of the fallopian tube. Int J Gynecol Obstet 1995;51(Suppl 1):S29-39.

[14] Kerin JF, Cooper JM, Price T, Herendael BJ, Cayuela-Font, Cher D, et al. Hysteroscopic sterilization using a micro-insert device: results of a multicentre Phase II study. Hum Reprod 2003;18(6):1223-30.

[15] Cooper JM, Carignan CS, Cher D, Kerin JF. Microinsert nonincisional hysteroscopic sterilization. Obstet Gynecol 2003;102(1):59-67.

[16] Alderman PM. Complications in a series of 1224 vasectomies. J Fam Pract 1991;33(6): 579-84.
[17] Kerin JF, Munday DN, Ritossa MG, Pesce A, Rosen D. Essure hysteroscopic sterilization: results based on utilizing a new coil catheter delivery system. J Am Assoc Gynecol Laparosc 2004;11(3):388-93.

[18] Mino M, Arjona JE, Cordon J, Pelegrin B, Povedano B, Chacon E. Success rate and patient satisfaction with the Essure sterilisation in an outpatient setting: a prospective study of 857 women. BJOG 2007;114(6):763-6.

[19] Levy B, Levie MD, Childers ME. A summary of reported pregnancies after hysteroscopic sterilization. J Minim Invasive Gynecol 2007;14(3):271-4.

[20] Levie MD, Chudnoff SG. Office hysteroscopic sterilization compared with laparoscopic sterilization: A critical cost analysis. J Minim Invasive Gynecol 2005;12(4): 318-22.

[21] Hopkins MR, Creedon DJ, Wagie AE, Williams AR, Famuyide AO. Retrospective cost analysis comparing Essure hysteroscopic sterilization and laparoscopic bilateral tubal coagulation. J Minim Invasive Gynecol 2007;14(1):97-102.

[22] Kerin JF, Cattanach S. Successful pregnancy outcome with the use of in vitro fertilization after Essure hysteroscopic sterilization. Fertil Steril 2007;87(5) 1212. e1-4.

[23] Kerin JF, Munday D, Ritossa M, Rosen D. Tissue encapsulation of the proxima Essure micro-insert from the uterine cavity following hysteroscopic sterilization. J Minim Invasive Gynecol 2007;14(2):202-4.

[24] Valle RF, Valdez J, Wright TC, Kenney M. Concomitant Essure tubal sterilization and Thermachoice endometrial ablation: feasibility and safety. Fertil Steril 2006;86(1):152-8.

[25] Thiel JA, Ciaran G. Placement of Essure device immediately following Novasure endometrial ablation. J Minim Invasive Gynecol 2006;13(5S):S104.

[26] Donnadieu AC, Fernandez $\mathrm{H}$. The role of Essure sterilization performed simultaneously with endometrial ablation. Curr Opin Obstet Gynecol 2008;20(4):359-63.

[27] Shavell VI, Abdallah AE, Diamond MP, Kmak DC, Berman JM. Post-Essure hysterosalpingography compliance in a clinic population. J Minim Invasive Gynecol 2008;15(4):431-4

[28] Weston G, Bowditch J. Office ultrasound should be the first-line investigation for confirmation of correct ESSURE placement. Aust N Z J Obstet Gynaecol 2005;45(4): 312-5.

[29] Kerin JF. Levy BS.Ultrasound: An effective method for localization of the echogenic Essure sterilization micro-insert: Correlation with radiologic evaluations. J Minim Invasive Gynecol 2005;12(1):50-4.

[30] Veersema S, Vleugels MP, Timmermans A, Brolmann HA. Follow-up of successful bilateral placement of Essure microinserts with ultrasound. Fertil Steril 2005;84(6): 1733-6.

[31] Vancaillie TG, Anderson TL, Johns DA. A 12-month prospective evaluation of transcervical sterilization using implantable polymer matrices. Obstet Gynecol 2008;112(6):1270-7.

[32] FDA Executive Summary. P070022 Adiana Transcervical Sterilization System. Prepared for the December 13, 2007 meeting of the Obstetrics \& Gynecology Devices Panel.

[33] Shavell VI, Abdallah ME, Shade Jr GH, Diamond MP, Berman JM. Trends in sterilization since the introduction of Essure hysteroscopic sterilization. J Minim Invasive Gynecol 2009;16(1):22-7. 